\title{
Back to the Future Consequences: Considerations of Future Consequences (CFC) Measure Correlates with Exercise Intensity
}

\author{
Calvin G. Isch \\ Faculty Mentors: Samuel A. Nordli, Department of Cognitive Science and Psychology, Indiana University \\ Bloomington, Peter M. Todd, Department of Cognitive Science and Psychology, Indiana University Bloomington
}

\begin{abstract}
What motivational and trait differences allow some individuals to achieve long-term goals where others fail? Activity in the pursuit of these long-term goals can provide both immediate and future rewards, though it often requires the sacrifice of short-term gratification for the pursuit of the goal. For instance, when a student studies for a test, she is not playing games with friends. Exercise is such an activity. Although individuals may gain immediate rewards from exercise, they often describe future goals (e.g. staying healthy or training for a competition) as partially motivating their behavior (Ebbem \& Brudzynski, 2008). Because exercise is an activity with future rewards, individual differences in future orientation may account for variance in exercise behavior. In this experiment, we use the Consideration of Future Consequences (CFC) questionnaire (Joireman et al., 2012) to measure future orientation, ask participants about perceived rewards associated with exercise, and record their behavior within a given workout. We find that CFC scores predict several aspects of exercise including frequency of engagement, intensity of exercise, and perceived future benefits associated with the activity-providing evidence that individuals who score highly on CFC believe exercise provides greater future benefits and exhibit increased activity to attain them.
\end{abstract}

KEYWORDS: future orientation, goal achievement, motivation, self control, exercise

\section{INTRODUCTION}

$\mathrm{P}$ eople who attempt to complete a college degree, train for a marathon, or quit a bad habit are pursuing long-term goals. While such goals are highly valued, people frequently fail to accomplish them: a US News report found that over eighty percent of New Year's Resolutions are given up by February (Luciani, 2015). Because these subjectively valuable goals are difficult to carry to fruition, it is important to understand the processes that lead to successes and failures in their pursuit.

Long-term goals require continual engagement in goal-directed activities and often involve present sacrifices with delayed benefits (Baumeister \& Tierney, 2012). Existing research provides support for several strategies to motivate an individual's pursuit of these goals. Some have found that attending to delayed rewards increases performance (Mischel et al., 1989), while others report that perceived immediate rewards serve as a stronger predictor of persistence (Woolley \& Fishback, 2016). In addition to the effects of perceived rewards, certain personality traits might also lead to increased goal-directed behavior. Specifically, some people report valuing the future more than others, a trait captured by the Consideration of Future Consequences (CFC) scale (Strathman et al., 1994). This paper explores the effects of perceived present and future rewards as well as CFC on several facets of goal-directed behavior as measured by college students' cardio-workout activity in a campus gym.

\section{Activity Engagement and Long-Term Goals}

Long-term goals require continued engagement in goal-directed activities. Such engagement can be split into different components. We address three facets of engagement in cardio-workout routines: frequency, duration, and intensity. Activity persistence, or the duration of an activity, is in part determined by perceived value gained relative to the costs of continuing with a certain action (Rothman, 2000). Similarly, frequency of engagement and intensity of activity may also be driven by this value-cost calculation. Activities with high value and low cost may generally be engaged with more often, for longer, with more intensity than those with relatively low value and high cost. Activities for long-term goals often have distant perceived rewards and immediate costs, and as a result, they generally require self-control.

\section{Self Control and Motivation}

Whether it be eating chocolate and giving up on a diet, excessively watching Netflix instead of studying, or participating in other immediately-gratifying behaviors at the expense of long-term goals, nearly everyone has experienced a failure of self-control. In contrast, effective self-control leads to improved performance on individual tasks, and people who routinely show self-control perform better on many social and academic outcomes, accomplishing more long-term goals (Mischel et al., 1989). There are several techniques one can implement to improve self-control, including many that focus on increasing the perceived value gained from a task and decreasing its costs (Duckworth et al., 2018). That self-control involves a cost-benefit calculation when considering how to engage with a given activity suggests that it is modulated by one's motivation for various outcomes (Berkman et al., 2017).

There are many theories on the mechanisms involved with motivation. From "fuel" limited accounts (Galliot et al., 2007) to mechanisms that balance immediate and future rewards (Kurzban et al., 2013), these models explain why we are limited in our ability to persist in activities with distant rewards that come at the expense of immediate gratification. According to each perspective, the persistence and intensity of engagement is dependent on the value that the individual places on the immediate and delayed outcomes associated with the task. Most activities involve both immediate and delayed rewards, and while people frequently claim that delayed rewards are critical for increasing activity engagement (Fishbach \& Choi, 2012), some scholars have found immediate rewards to be more salient at driving behavior (Woolley \& Fishbach, 2016). For example, a student's interest in a subject, a characteristic that implies more immediate rewards associated with the activity, motivates their academic engagement and achievement with that subject (Harackiewicz et al., 2016). With evidence indicating both types of rewards are useful and valued with exercise (Ebbem \& Brudzynski, 2008), there may be trait differences among individuals that allow certain types of rewards (e.g. future rewards) to be more effective at motivating behavior for some than others. 


\section{Considerations of Future Consequences Scale}

Some individuals report valuing future rewards more highly, frequently allowing future goals to drive their day-to-day behavior. Others report living more in the present, expecting the future to take care of itself. To measure the differences in future considerations between individuals, a survey-based scale was developed: the Consideration of Future Consequences scale (Strathman et al., 1994). Past work has demonstrated that high scores on the CFC scale predict higher frequency of healthrelated behavior, reduced aggression, increased dietary control, and more frequent exercise behavior (Joireman et al., 2003; Piko \& Brassai, 2009; Adams \& Nettle, 2009). Subsequent analysis suggests there is evidence for two factors within the scale: CFC-future (CFC-F) and CFCimmediate (CFC-I) (Joireman et al., 2012). CFC-F captures a tendency to focus on the future consequences of engaging in certain behavior; CFC-I measures a tendency to focus on the immediate consequences of one's actions or the current opportunities missed. The distinction can be important for determining the motivators driving actions. For example, past work has found that those who exercise less often are concerned with the immediate consequences of their behavior, and prefer other activities to exercise, though they may still care about the future benefits associated with exercise (Joireman et al., 2012).

To investigate the connection between CFC scores and factors associated with long-term goal pursuit, we looked at college students' exercise behavior. We collected data to address three hypotheses: (1) CFC score will correlate with workout frequency, persistence, and intensity, (2) CFC will correlate with the magnitude of future rewards that participants perceive themselves to gain through working out, and (3) participants' perceived magnitude of immediate and future rewards will predict workout frequency, persistence, and intensity. These three hypotheses are related through the process outlined above: high-CFC individuals focus more on future goals, thus increasing their motivation for future rewards and consequently improving self-control to continue with tasks that lead to the accomplishment of those aims.

\section{METHODS}

\section{Participants}

We invited 150 individuals in an on-campus gym to complete pre- and post-workout surveys in return for a nutrition bar and a Gatorade. Participants were approached before starting a workout on a cardio machine. 20 participants did not return for the post-workout survey, and were excluded from analysis, leaving 130 remaining participants (71 females; $\mathrm{M}$-age $=21.4 ; \mathrm{SD}=5.6$ ). IRB exemption was granted for this experiment, and subjects were provided information on the study (including procedures and voluntary participation) before agreeing to participate.

\section{Procedure}

Participants first answered questions addressing how important the immediate, delayed, and distant rewards associated with their workout were to them (all questions are included in Appendix 1). Immediate rewards refer to those realized during the workout, delayed rewards occur within a few months, and distant rewards are the impacts of the exercise on health in five years' time. Participants answered these questions on a 1-6 Likert scale. Throughout the rest of the paper we refer to their answers on these questions as reward-importance score. Participants then answered questions on their weekly exercising habits, larger goals regarding exercise, anticipated workout length, and questions about whether that day's exercise activity was part of an existing routine. Next, they completed the 14-question CFC scale, with responses ranging from 1-7. Once the survey was completed, experimenters gave participants an identification card, noted down the time, and instructed them to begin their workout and record the information provided by their cardio machine (either with their phone's camera or on a supplied card) once they were finished.

After completing their workout, participants returned to the experimenters and filled out another short survey. They reported the machine used as well as the duration, distance, and caloric information provided by their cardio machines. Participants then answered three questions addressing their perceptions of the amount of immediate, delayed, and distant rewards gained through their workout. We refer to these as reward-magnitude scores throughout the paper. Finally, participants answered demographic questions before receiving their snack.

\section{RESULTS}

Although workouts are not differentiated by machine in our analyses, participants used a number of different exercise machines in this study, with the elliptical being most common $(27.7 \%, \mathrm{n}=36)$. The bike and the treadmill were each used by another $16.2 \%(n=21$ each) of participants, $13.8 \%(\mathrm{n}=18)$ choose the stepping machine, and the remaining $26.1 \%(n=34)$ exercised on other machines (curve, $n=8$; rowing machine, $\mathrm{n}=4$; track, $\mathrm{n}=3$; and multiple machines, $\mathrm{n}=19$ ). All machines (except the track) reported number of calories burned.

\section{Calculating CFC}

Of the 14 questions on the CFC scale, seven target CFC-I and seven target CFC-F. We used these scales independently and combined them together into an overall CFC score by reverse coding CFC-I responses and summing those answers with the CFC-F results.

To determine the relationship between CFC and frequency, persistence, and intensity of working out, we ran Pearson Correlations on overall CFC score $(\mathrm{M}=70.5, \mathrm{SD}=10.1)$ with reported weekly visits $(\mathrm{M}=4.1, \mathrm{SD}=1.6)$, minutes spent exercising $(\mathrm{M}=41.2, \mathrm{SD}=20.9)$, and workout intensity $(\mathrm{M}=8.5, \mathrm{SD}=4.8)$. We calculated intensity by dividing participants' reported number of calories burned by the number of minutes spent exercising. If participants used multiple machines, the calories burned on each machine were summed prior to dividing by total time. Seventeen participants were excluded from this calculation, as they failed to report the number of calories burned, resulting in 113 participants in the intensity calculation. There was no significant correlation between $\mathrm{CFC}$ and time spent exercising $(\mathrm{R}=$ $-.03, \mathrm{p}=.74)$, but there were significant correlations between $\mathrm{CFC}$ and intensity $(\mathrm{R}=.19, \mathrm{p}=.048)$ as well as between $\mathrm{CFC}$ and frequency of reported weekly workouts $(\mathrm{R}=.38, \mathrm{p}<.01)$. A scatterplot and best-fit line of participants' reported number of weekly workouts and CFC scores is shown in Figure 1.



Figure 1.

CFC related to Weekly Workouts. 
Additionally, participants' CFC scores were correlated with the reported duration (in months) of their existing exercise routines (M $=12.9, \mathrm{SD}=19.3, \mathrm{R}=.18, \mathrm{p}=.04$ ), as well as reported intention to start a new routine or continue an existing routine on a 6-point Likert scale $(\mathrm{M}=5.4, \mathrm{SD}=1.0, \mathrm{R}=.43, \mathrm{p}<.001)$.

To examine how $\mathrm{CFC}$ related to the different perceived rewards, we ran Pearson correlation analyses on overall CFC and participants' reported reward-importance (i.e. how important each reward type was to them) and reward-magnitude (i.e. how effective the workout was at producing each reward) scores for their workout. Table 1 shows the results. There are significant, positive correlations between CFC and reported delayed and distant reward-importance and reward-magnitude scores, but no statistically significant relationship between CFC and immediate rewards.

\begin{tabular}{lccc}
\hline \multicolumn{1}{c}{ Rewards } & Mean (SD) & $\mathrm{N}$ & $\begin{array}{c}\text { Pearson Correlation, } \\
\text { Significance }\end{array}$ \\
\hline Immediate-importance & $4.6(1.2)$ & 130 & $\mathrm{R}=.01, \mathrm{p}=.95$ \\
Delayed-importance & $5.5(0.7)$ & 130 & $\mathrm{R}=.25, \mathrm{p}<.01$ \\
Distance-importance & $5.3(1.0)$ & 130 & $\mathrm{R}=.33, \mathrm{p}<.001$ \\
Immediate-magnitude & $4.5(1.1)$ & 130 & $\mathrm{R}=.10, \mathrm{p}=.24$ \\
Delayed-magnitude & $5.0(1.0)$ & 130 & $\mathrm{R}=.18, \mathrm{p}=.04$ \\
Distance-magnitude & $5.0(1.1)$ & 130 & $\mathrm{R}=.38,=<.001$ \\
\hline
\end{tabular}

Table 1.

Relation of CFC to reward-importance and magnitude.

Reward-importance and magnitude scores are pulled from participant responses to the questions asking how important they considered the immediate, delayed, and distant rewards associated with working out and how much they perceived their workout as rewarding on each account. Responses were given on a 6-point Likert scale. Bolded items have $p<.05$ Significance

To address how perceived rewards predicted each aspect of exercise activity, we ran standard multiple regression analyses with the three reward-magnitude scores serving as predictor variables for each component of exercise activity (intensity, duration, and frequency). This resulted in three multiple regressions with reported immediate, delayed, and distant rewards predicting a different aspect of exercise in each regression. These analyses show how each reward predicts exercise intensity, duration and frequency when controlling for the other perceived rewards. Table 2 shows the beta coefficients of each predictor variable for each equation.

\begin{tabular}{cccc}
\hline & $\begin{array}{c}\text { Duration } \\
(\mathrm{n}=130)\end{array}$ & $\begin{array}{c}\text { Frequency } \\
(\mathrm{n}=130)\end{array}$ & $\begin{array}{c}\text { Intensity } \\
(\mathrm{n}=113)\end{array}$ \\
\hline $\begin{array}{c}\text { Immediate-magnitude } \\
\text { Delayed-magnitude }\end{array}$ & $\begin{array}{c}\beta=.06, \mathrm{p}=.53 \\
\boldsymbol{\beta}=. \mathbf{2 1}, \mathbf{p}=. \mathbf{0 5}\end{array}$ & $\begin{array}{c}\beta=.01, \mathrm{p}=.93 \\
\beta=.02, \mathrm{p}=.83\end{array}$ & $\begin{array}{c}\beta=-.18, \mathrm{p}=.11 \\
\beta=.13, \mathrm{p}=.29\end{array}$ \\
$\begin{array}{c}\boldsymbol{\beta}=-.15, \mathrm{p}=.12 \\
\boldsymbol{\beta}=\mathbf{. 3 5}, \mathbf{p}<. \mathbf{0 1}\end{array}$ & \begin{tabular}{c}
$\beta=-.03, \mathrm{p}=.76$ \\
\hline
\end{tabular}
\end{tabular}

Table 2.

Multiple regression beta coefficients for each measure of exercise activity.

Immediate, delayed, and distant reward magnitudes are the predictor variables.

Bolded items have $p \leq .05$ Significance

When we regressed time spent exercising on the three rewards $\left(\mathrm{r}_{\text {immediate,delayed,distant }}=.230, \mathrm{p}=.076\right)$, we find that the delayed rewardmagnitude was the only significant predictor of duration $(\beta=.21$, $\mathrm{t}(130)=2.0, \mathrm{p}<.05)$. In other words, the more subjects believed that the exercise was beneficial at keeping them in shape (a delayed reward) the longer they exercised in a single visit. In our regression of exercise frequency on perceived rewards $\left(\mathrm{r}_{\text {immediate,delayed,distant }}=.357, \mathrm{p}<\right.$ .01 ) we find that distant reward-magnitude score (e.g. health in five years' time) was a significant predictor of the number of reported weekly workouts $(\beta=.346, \mathrm{t}(130)=3.8, \mathrm{p}<.01)$. Experienced immediate rewards did not predict duration or frequency of exercise with significance. Finally, when we regressed our intensity measure on the three perceived reward types $\left(\mathrm{r}_{\text {immediate,delayed,distant }}=.162, \mathrm{p}=.43\right.$ ), we found that no perceived reward type significantly predicted intensity.

\section{CFC Subscales}

To examine the relative contribution of the immediate and future $\mathrm{CFC}$ sub-scales, we ran Pearson correlations for both CFC-I, CFC-F, and all of the reward-importance and reward-valuation scores. Surprisingly, CFC-I showed no significant correlation with immediate rewards and had a negative correlation with ratings of distant reward-importance $(\mathrm{R}=-0.19, \mathrm{p}=.031)$. This trend continued for reward-magnitude scores, with the perceived efficacy of a workout driving future outcomes decreasing with CFC-I $(\mathrm{R}=-.23, \mathrm{p}<.01)$. In contrast, CFC-F was positively correlated with delayed and distant rewardimportance scores (R-delayed $=.27$, R-distant $=.34, \mathrm{p}<.01$ for both) as well as the delayed and distant reward-magnitude scores (R-delayed $=.25, \mathrm{R}$-distant $=.37, \mathrm{p}<.01$ for both). Put simply, as CFC-F increased so did the reported importance of future rewards and reported beliefs about how an exercise would lead to those future outcomes. We also ran correlations between CFC-I, CFC-F and the three dimensions of goal directed activity. The results are presented in Table 3.

\begin{tabular}{lccccc}
\hline $\begin{array}{c}\text { CFC-I \& } \\
\text { Intensity }\end{array}$ & $\begin{array}{c}\text { CFC-I \& } \\
\text { Persistence }\end{array}$ & $\begin{array}{c}\text { CFC-I \&Weekly } \\
\text { Workouts }\end{array}$ & $\begin{array}{c}\text { CFC-F \& } \\
\text { Intensity }\end{array}$ & $\begin{array}{c}\text { CFC-F \& } \\
\text { Persistence }\end{array}$ & $\begin{array}{c}\text { CFC-F \&Weekly } \\
\text { Workouts }\end{array}$ \\
\hline $\mathrm{R}=-.21$, & $\mathrm{R}=-.05$, & $\mathrm{R}=-.20$, & $\mathrm{R}=.04$, & $\mathrm{R}=-.01$, & $\mathrm{R}=.41$ \\
$\mathrm{p}=.017$ & $\mathrm{p}=.52$ & $\mathrm{p}=.022$ & $\mathrm{p}=.66$ & $\mathrm{p}=.94$ & $\mathrm{p}<.01$ \\
\hline
\end{tabular}

Table 3.

$C F C-I$ and $C F C$-F related to workout activity measures.

Bolded items have $p<.05$ Significance

\section{DISCUSSION}

This study demonstrates how CFC relates with several aspects of exercise-an activity with many delayed benefits. Our results are consistent with previous findings that demonstrate the relationship between CFC and the frequency of engagement in present activity with delayed rewards (Strathman et al., 1994). Additionally, we find that CFC correlates with workout intensity within a single session; high-CFC individuals work out with more vigor compared to those low in CFC. These outcomes may be driven by the ways those high in $\mathrm{CFC}$ view the rewards associated with the activity. Our results provide evidence that these individuals report that they both value the associated future rewards more and believe that the workout is more effective at bringing about those future aims. These findings applied to typical accounts of motivation theory imply that high-CFC individuals are more highly motivated to pursue tasks with future benefits, allowing them to more easily exert self-control and engage with the activity more often with greater intensity.

Our results show no significant relationship between perceived immediate rewards and various aspects of workout performance; instead we find that perceived delayed rewards are better predictors of exercise activity. As such, our findings seem to conflict with earlier studies that found that immediate rewards serve as a better predictor of persistence than delayed rewards (Woolley \& Fishbach, 2016). A 
methodical difference in study design may explain this discrepancy. Unlike in the experiment by Woolley and Fishbach, participants in our study answered survey questions before beginning their workout. Consequently, participant awareness of being observed and of study questions regarding workout goals and long-term rewards may have primed some participants to consider what they "ought" to do and to work harder than individuals working out without awareness of these study-specific factors. Subsequent research should avoid this confound by waiting to collect information on goals and future orientation until after the workout and presenting a cover story (e.g. "We are trying to understand why students go to the gym and what machines they use.") for any information collected before participants begin their workout.

Future work can expand upon these findings in many ways. First, it can measure the variables of interest from this study in different settings. The present study was limited to individuals who happened to be in a campus gym during the evening that the experiment took place, a group that might demonstrate different tendencies than the general population. Furthermore, our measures of some aspects of activity engagement (e.g., weekly workout frequency) are limited in that they are self-reported and are thus potentially influenced by imperfect recall, social desirability bias, etc. Future studies can directly measure activity over time to address this issue. By examining the relationship between CFC, the importance and magnitude of rewards, and activity performance, we will have a better understanding of the generalizability of these findings.

\section{CONCLUSION}

In summary, this experiment provides evidence that the CFC scale is a useful measure in understanding individual differences in exercise behavior, an activity with many future benefits. Gymgoers who score higher on the CFC scale workout more frequently, with greater intensity, and have a greater desire to create or continue a workout routine. They also rate future rewards as being more important and report that their current activity is more likely to drive future outcomes, a finding in line with typical explanations of motivation and self-control theory.

These findings provide evidence for individual differences that lead to varying amounts of activity directed toward future goals. Focusing on the future seems to increase goal salience and subsequent performance for tasks that involve many perceived future benefits. Using these results, individuals can attend to future rewards, find information that increases the amount they see a task as leading to that future aim, and ultimately improve their ability to achieve their long-term goals.

\section{ACKNOWLEDGEMENTS}

The authors thank Nick Riina, Nicholas Beuhler, and Jack Mosk for their help with data collection. They also thank Chris Geary and the staff of IU Recreational Sports for their assistance in providing a location for the study. Finally, they thank Kaitlin Woolley for her discussion and support.

\section{REFERENCES}

Adams, J., \& Nettle, D. (2009) Time perspective, personality and smoking, body mass, and physical activity: an empirical study. British Journal of Health Psychology, 14(1), 83-105.

Baumeister, R.F., \& Tierney, J. (2012). Willpower: Rediscovering the greatest human strength. New York, NY: Penguin Press.

Berkman, E.T., Hutcherson, C. A., Livingston, J.L., Kahn, L.E., Inzlicht, M. (2017). Self-control as value-based choice. Association for Psychological Science. 26(5), 422-428.
Duckworth, A. L., Milkman, K.L., \& Laibson, D. (2018) Beyond willpower: Strategies for reducing failures of self-control. Psychological Science in the Public Interest, 19(3), 102-129.

Ebbem, W. \& Brudzynski, L. (2008) Motivations and barriers to exercise among college students. Journal of Exercise Physiology. 11(5),1-11.

Fishbach, A., \& Choi, J. (2012). When thinking about goals undermines goal pursuit. Organizational Behavior and Human Decision Processes, 118, 99-107.

Galliot, M. T., Beumeister, R. F., DeWall, C. N., Maner, J. K., Plant, E. A., Tice, D. M., ... \& Schmeichel, B. J. (2007) Self-control relies on glucose as a limited energy source: Willpower is more than a metaphor. Journal of personality and social psychology, 92(2), 325-336.

Harackiewicz, J.M., Smith, J.L., \& Priniski, S.J. (2016) Interest matters: The importance of promoting interest in education. Policy Insights from the Behavioral and Brain Sciences, 3(2), 220-227.

Joireman, J., Anderson, J., \& Strathman, A. (2003). The aggression paradox: Understanding links among aggression, sensation seeking, and the consideration of future consequences. Journal of Personality and Social Psychology, 84(6), 1287-1302.

Joireman, J., Shaffer, M. J., Balliet, D., \& Strathman, A. (2012) Promotion orientation explains why future-oriented people exercise and eat healthy: Evidence from the two-factor consideration of future consequences-14 scale. Personality and Social Psychology Bulletin, $38(10), 1272-1287$.

Kurzban, R., Duckworth, A., Kable, J., \& Myers, J. (2013) An opportunity cost model of subjective effort and task performance. Behavioral and Brain Sciences, 36, 661-726.

Luciani J. (Dec. 29, 2015) Why 80 percent of new year's resolutions fail. U.S. News. Retrieved from: https://health.usnews.com/healthnews/blogs/eat-run/articles/2015-12-29/why-80-percent-of-newyears-resolutions-fail

Mischel, W., Shoda, Y., \& Rodriguez, M. I. (1989). Delay of gratification in children. Science, 244, 933-938.

Piko, B.F. , \& Brassai, L. (2009) The role of individual and familial protective factors in adolescents' diet control. Journal of Health Psychology, 14(6), 810-819.

Rothman, A. J. (2000). Toward a theory-based analysis of behavioral maintenance. Health Psychology, 19(1, Suppl.), 64-69.

Strathman, A., Gleicher, F., Boninger, D. S., \& Edwards, C. S. (1994) The consideration of future consequences: Weighing immediate and distant outcomes of behavior. Journal of Personality and Social Psychology, 66(4), 742-752.

Woolley, K., \& Fishback, A. (2017) Immediate rewards predict adherence to long-term goals. Personality and Social Psychology Bulletin, 43(2), 1-12. 


\section{APPENDIX I}

\section{Please answer the following questions:}

How important is it that your exercise today is enjoyable and fun? Not at all important

$$
2
$$

3

4

Very Important

How important is it that your exercise today is useful and effective at keeping you in shape?
Not at all important
1
2
3
4
Very Important

How important is it that your exercise today contributes positively to your health in 5 years?

Not at all important

$\begin{array}{llllll}1 & 2 & 3 & 4 & 5 & 6\end{array}$

In one week, how many times do you typically work out? times

In one setting, how long do you usually workout? minutes / Until satisfied.

How long do you plan to work out today? minutes / Until satisfied.

Do you have another activity limiting how long you can stay today? Yes, in mins / No

Do you have a larger goal in visiting the gym? Circle all that apply.

Stay fit Training for a competition in the next 3 months

Lose weight

Training for a competition over 3 months away

Get in shape Be with friends

Look good for winter break

Health reasons

Look good for spring break

Other

Do you come to the gym regularly? If so, how long have you had a routine?

Yes / No months

Do you come in on the same days each week?

Yes / No

Do you intend to create (or continue) a routine of going to the gym over the next few months?

No Intention

12 2 3 4
Strong Intention 5

\section{Considerations of Future Consequences Scale}

For each of the statements shown, please indicate whether or not the statement is characteristic of you. If the statement is extremely uncharacteristic of you (not at all like you) please write a " 1 " in the space provided to the left of the statement; if the statement is extremely characteristic of you (very much like you) please write a " 7 " in the space provided. And, of course, use the numbers in the middle if you fall between the extremes.

$1=$ Not at all like you $7=$ very much like you

I consider how things might be in the future, and try to influence those things with my day to day behavior.

Often I engage in a particular behavior in order to achieve outcomes that may not result for many years.

I only act to satisfy immediate concerns, figuring the future will take care of itself.

My behavior is only influenced by the immediate (i.e., a matter of days or weeks) outcomes of my actions.

My convenience is a big factor in the decisions I make or the actions I take.

I am willing to sacrifice my immediate happiness or wellbeing in order to achieve future outcomes.

I think it is important to take warnings about negative outcomes seriously even if the negative outcome will not occur for many years.

I think it is more important to perform a behavior with important distant consequences than a behavior with less important immediate consequences.

I generally ignore warnings about possible future problems because I think the problems will be resolved before they reach crisis level.

I think that sacrificing now is usually unnecessary since future outcomes can be dealt with at a later time.

I only act to satisfy immediate concerns, figuring that I will take care of future problems that may occur at a later date.

Since my day to day work has specific outcomes, it is more important to me than behavior that has distant outcomes.
When I make a decision, I think about how it might affect me

How much do you intend to have a routine of going to the gym in one year?

No Intention

Strong Intention

1

3

My behavior is generally influenced by future consequences. 


\title{
Information about Workout and Demographics
}

Info about your workout:

Machine (circle one):

Elliptical

Treadmill

Rowing

Machine

Bicycle

Stepping machine Minutes

Other

Duration:

Distance:

Calories:

\author{
Distance:
}

How enjoyable or fun did you find your workout today?

Not at all enjoyable

1

2

3

4

Very enjoyable

How useful and effective was your workout for keeping/getting you in shape?

Not at all useful

12

3

4

Very useful

How much will your exercise today positively impact your health five years from now?

Not at all impactful

2

3

4

Very impactful

What is your Gender?

What is your age? 